\title{
Medidas para reforzar la unión económica y monetaria
}

Measures to reinforce the economic monetary union

\section{Resumen}

El presente trabajo de investigación recopila algunas de las diferentes medidas tomadas en los últimos años como consecuencia de los retos y complicaciones a los que la Economía Europea se ha enfrentado; retos que hasta la fecha, siguen presentes en la constante búsqueda para reforzar y mejorar las políticas económicas y monetarias de la UE. Si bien las restricciones y luchas en la UE (tanto externas como internas), no sólo se limitan a aspectos económicos y monetarios, lo cierto es que éstos han tomado en la última década, mayor importancia e inversión de tiempo y esfuerzo por parte de los diferentes gobiernos participantes, como consecuencia del contagio de la crisis financiera que se originó en Estados Unidos. Este trabajo se centra en explicar las acciones que se instrumentaron y que se refieren específicamente a la Gobernanza Económica y en donde se trata de contestar la pregunta de qué reforzar, por qué y qué medidas se tomaron para ello, entre las que se encuentran detalladas políticas económicas como el "Two Pack" y el "Six Pack". Finalmente se ofrecen conclusiones y recomendaciones sobre el tema en donde se advierte de las posibles consecuencias y nuevos retos a los que la UE se podría enfrentar de no tener éxito con la dirección tomada hasta ahora.

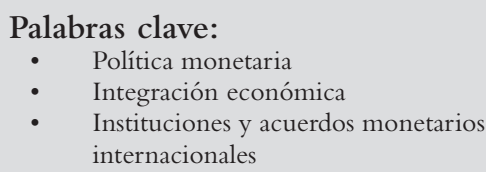

\begin{abstract}
The present research work collects some of the different measures taken in recent years as a result of the challenges and complications the European economy has faced to date, challenges that are still present in the constant search of ways to strengthen and improve the EU's economic and monetary policies. While restrictions and conflicts in the EU (both external and internal), are not only limited to economic and monetary aspects of the Union, the fact is that they have taken greater importance in the past decade, investing a considerable amount of time and effort on the part of the participating Governments, especially after the financial crisis that started in the United States. This work focuses on explaining the actions that followed, which relate specifically to Economic Governance and where the focus is answering the key questions of "what, why and what" measures were Finally the work offers conclusions and recommendations on the subject and warns of possible consequences and challenges the EU could face if it does not succeed with the direction taken so far.
\end{abstract}

Santiaga Ánima Puentes* JEL: E52, F15, F33

Keywords:

Monetary Policy

Economic Integration

International Monetary Arrangements and Institutions

\section{Introducción}

En la última década y como resultado de los diversos retos que la Unión Europea (UE) ha enfrentado a nivel económico, las medidas para reforzar la Unión Económica y Monetaria en la UE se han incrementado marcadas por el aumento en su complejidad y frecuencia.

\footnotetext{
* Profesora Titular de la Materia de Economía Europea y Estructura Económica Mundial Actual en la Facultad de Economía, UNAM. Este ensayo se elaboró como documento final del curso sobre la Unión Europea realizado en la estancia semestral sabática en Madrid, España, ofrecido por la Escuela Diplomática de Madrid. <animas@economía.unam.mx>
} 
Son claros los esfuerzos que se han hecho en todos los campos en esta región para resolver los problemas, -que por la interconexión del sistema bancario mundial y unas regulaciones bancarias "relajadas"-, como efecto de la globalización aflora la crisis originada en Estados Unidos antes de terminar la primera década del siglo xxI, cuando justo al iniciar el nuevo milenio la economía europea parecía que tenía muchas razones para ser optimista acerca de sus perspectivas de convertirse en un verdadero motor de crecimiento. ${ }^{1}$ "Entre 2002 y 2008 aumento el crédito de forma irresponsable gestando el preludio de una grave recesión en las economías maduras, que primero afecto a la economía estadunidense y rápidamente se extendió a Europa" (Aldcroft, 2012, p. 453).

Poco a poco se fueron aceptando las causas que pusieron en peligro al euro ubicadas por varios especialistas en la gestación misma de la política monetaria, de modo que la actuación de autoridades e instituciones correspondientes en la UE y en la Euro Zona (EZ) fueron asumiendo una serie de respuestas para tratar de combatirla y al mismo tiempo lograr el crecimiento económico para disminuir el alto desempleo que iba aumentando en varios países de manera alarmante. $^{2}$

En este ensayo se abordarán las acciones que se instrumentaron y que se refieren específicamente a la Gobernanza Económica. ${ }^{3}$

¿Qué reforzar y por qué?

Para apuntalar a la Unión Económica y Monetaria (UEM) se reforzaron dos de sus pilares en el periodo 2011-2014:

1) el de disciplina presupuestaria, reforzando el pacto de estabilidad y crecimiento $(\mathrm{PEC})^{4} \mathrm{y}$

2) el de coordinación de Políticas económicas (GOPES) para sanear las cuentas públicas; por el aumento tan significativo que experimento el déficit y la deuda

\footnotetext{
${ }^{1}$ Estas perspectivas se anuncian en la famosa Agenda 2000.

${ }^{2}$ El caso de Grecia y España sobre todo.

${ }^{3}$ El objetivo de la gobernanza económica es potenciar una mayor coordinación de políticas económicas con objeto de impulsar el crecimiento y la creación de empleo en el futuro.

${ }^{4}$ El Pacto de Estabilidad y crecimiento (PEC) fue creado en 1997 para dar paso a la $3{ }^{\text {a }}$. Fase de la UEM y coordinar las políticas presupuestarias nacionales. Su objetivo principal era el mantenimiento de unas finanzas públicas sanas, es decir un déficit máximo de 3\% y una deuda de $60 \%$ /PIB. En la práctica se revelo como un mecanismo insuficiente e incapaz de lograr en forma efectiva esos objetivos. Las recomendaciones de las autoridades europeas a que daba lugar, la presión de los otros países y aun el procedimiento de déficit excesivo, en su primera versión, no constituyeron un mecanismo que ofreciera en la práctica resultados satisfactorios.
} 
de varios países miembros ${ }^{5}$ que obligaron a la UE a introducir elementos estabilizadores para tratar de corregirlos.

En el marco legislativo, tuvieron lugar 3 acciones concretas, denominadas:

1. Six Pack (diciembre, 2011): refuerza el PEC e introduce un procedimiento para controlar desequilibrios macroeconómicos (PDM).

2. Two Pack (2013): introduce la evaluación de presupuestos nacionales y da mayor seguimiento al cumplimiento del PEC.

3. Tratado de Estabilidad, Coordinación y Gobernanza (TECG) 2013, incluye Pacto Fiscal.

1) Su denominación de "Six pack" obedece a que está constituido por seis Normas, cinco Reglamentos y una Directiva, que entraron en vigor en diciembre de 2011. Constituye un conjunto normativo de aplicación en toda la UE, sin perjuicio de tener disposiciones específicas, en particular relativas a sanciones, de aplicación exclusiva a los países de la EZ. Este paquete regulatorio no solo establece sistemas de vigilancia de las políticas fiscales en sentido estricto, sino que alcanza a las magnitudes macroeconómicas bajo la forma de un nuevo Procedimiento de Desequilibrios Macroeconómicos (nPDM).

- En el ámbito fiscal, la regulación que incorpora este paquete implica un refuerzo del sistema de control que en este ámbito suponía el PEC pero intentando superar sus carencias. Así, el saldo presupuestario de los EM debe ir convergiendo a una cifra señalada, configurada para cada país como un objetivo a mediano plazo.

- Adicionalmente, la cifra de déficit no debe exceder 3\% y la deuda $60 \%$ del РIB. De este modo, el six pack refuerza no solo el aspecto de medidas preventivas de convergencia fiscal del PEC sino también las medidas correctoras, con un nuevo procedimiento de Déficit Excesivo (nPDex) que se pone en marcha cuando no se cumplen los objetivos de déficit o deuda.

- En el ámbito de las medidas preventivas el refuerzo se produce por la vía de la mayor precisión en la definición de los objetivos a alcanzar, así como de lo que constituye una "desviación significativa" en su camino de cumplimiento. Respecto de las cifras de convergencia del objetivo a mediano plazo de cada país y de las comprometidas en el ajuste podría iniciarse un procedimiento de Déficit Excesivo por no lograrse el ritmo de cumplimiento de la evolución de la deuda pública al nivel de 60\%/PIB, a diferencia de en la versión anterior, en el que solo cabía por incumplimiento del nivel de 3\% de déficit.

\footnotetext{
${ }^{5}$ Grecia, Irlanda, Portugal y después Chipre, España y actualmente Italia, Francia...
} 
- En el ámbito de las medidas de corrección y sanciones, se refuerza la posibilidad de imponer sanciones a los EM, tanto en la fase de prevención como en las diferentes fases del Procedimiento de Déficit Excesivo, sanciones que podrían llegar a alcanzar 0.5\% del PIB del Estado incumplidor, facilitando su imposición respecto al régimen anterior al haber cambiado, para la mayoría de las sanciones, y especialmente para los países de la ZE, el sistema de decisión para su imposición: se pasa de un sistema de adopción por mayoría a otro, denominado "de votación por mayoría cualificada inversa”, en el que la propuesta de la Comisión se considera adoptada por el Consejo salvo que una mayoría de países vote en contra de ella.

2) En 2013 se introduce el denominado "Two pack" formado por dos Reglamentos comunitarios que entraron en vigor el 30 de mayo, aprobados en el ámbito del artículo 136 del Tratado de Funcionamiento de la Unión Europea (TFUE), aplicables únicamente al área del euro.

Su objetivo es el refuerzo de la coordinación y vigilancia de las políticas presupuestarias nacionales, que incluyen un proceso de evaluación de los proyectos de presupuestos de los países, previo a su aprobación.

Además de establecer normas y objetivos presupuestarios comunes a nivel nacional supervisados por autoridades independientes, adicionadas a las previsiones del PEC, se obliga a los EM a presentar Programas de Estabilidad o de Convergencia ante la Comisión y el Consejo en su reunión de Primavera, líneas básicas de sus planes de previsión financiera a mediano plazo, así como un ejercicio de otoño para una más estricta supervisión de sus proyectos de presupuestos. La Comisión analiza si el proyecto de presupuestos está en línea con las recomendaciones del Semestre Europeo y con las del PEC y si estima que no se ajusta, puede llegar a pedir que se revise sin perjuicio de realizar otras observaciones que serían discutidas en las reuniones del eurogrupo.

Aunque los Parlamentos nacionales conservan su soberanía para aprobar las leyes de presupuestos, se incrementa la vigilancia y la presión de los otros EM en este ámbito, y se adicionan a los antecedentes a tomar en cuenta para la aprobación tanto de los informes de la Comisión como los de la Autoridad independiente de Responsabilidad Fiscal.

Por otra parte, se refuerzan las medidas a adoptar en el caso de países que estén en situación de dificultades financieras, en esos casos la Comisión puede acordar una vigilancia reforzada, que implicara la obligatoria adopción por los Estados de medidas de corrección de las causas de la inestabilidad, también hará misiones periódicas de supervisión para comprobar los avances, y pedirá de manera obligatoria información más detallada y desagregada, dando cuen- 
ta de los avances, en forma trimestral al Eurogrupo. Asimismo, la Comisión prestará asistencia técnica para diseñar planes de vigilancia y corrección de los desequilibrios y desajustes a los países.

En caso de incumplimiento de estos programas, el Consejo podría restringir las ayudas comprometidas con el país. En estas situaciones, los diferentes elementos ordinarios de coordinación y vigilancia quedan desplazados por el concreto programa del país. Una vez superada la situación y cumplido el programa, se establecen no obstante medidas de seguimiento y monitorización, al menos hasta que $75 \%$ de la deuda del mismo se haya sufragado.

La legislación sobre supervisión presupuestaria conocida como Two pack se aplicó por primera vez en la elaboración de los planes presupuestarios de 2014 en los estados que integran la EZ.

Según establecen los dos reglamentos comunitarios que componen el paquete, los estados de la EZ deben enviar a la Comisión los presupuestos nacionales antes de su aprobación por los parlamentos de cada país, de tal manera que si Bruselas detectara un "incumplimiento serio" del PEC podría requerir una propuesta revisada. Y si el Estado incumpliera este último requerimiento, la Comisión podría notificar esta circunstancia al eurogrupo, quien podría iniciar un debate al respecto. El pasado 15 de noviembre la Comisión hizo públicos sus dictámenes.

La Coordinación de política económica se reforzó sobre todo mediante:

1. Tratado de Estabilidad, Coordinación y Gobernanza del euro (TECG) ${ }^{6}$

2. Pacto por un EURO plus

3. Semestre europeo

4. Se trata de un tratado entre estados y no de una norma comunitaria, por lo que en algunos aspectos es coincidente y su aplicación correrá paralela a la del Six pack.

Su entrada en vigor estaba condicionada a la ratificación de al menos 12 estados de la EZ, habiendo sido firmado por 25 de los 27 EM (todos, excepto la Republica Checa y Reino Unido, el 25 de marzo de 2012). En todo caso, será únicamente obligatorio para los países del euro; para el resto de firmantes solo

\footnotetext{
${ }^{6}$ Por primera vez en la historia del largo proceso de integración europea se prescindió del requisito de unanimidad para la entrada en vigor de un Tratado. En su lugar se introdujo la regla de "casi 3/4 (en el caso de los 17 países miembros de la eurozona (EZ) solo 12 eran necesarios para ratificar el Tratado además de no considerar a los 10 EM que no pertenecen la Ez.).
} 
lo será en tanto que se incorporen al euro o si así lo deciden expresamente (en cuyo caso pueden decidir que partes les serian de aplicación).

La parte de medidas fiscales del tratado se conoce como el "Pacto fiscal".

Los países sujetos a su aplicación vienen obligados a cumplir criterios de convergencia, definidos, como en el Six pack, por referencia a un objetivo específico a mediano plazo para cada uno de ellos, en términos de PIB es más estricto que en las normas anteriores, cifrado en $0.5 \%$ de déficit estructural (excluidas medidas excepcionales y ajustes cíclicos), que podría llegar a 1\% en aquellos Estados con niveles de deuda pública muy por debajo del objetivo de 60 por ciento.

En caso de incumplimiento del objetivo, o de la senda predeterminada para la convergencia, se establecen mecanismos de corrección que se ponen en marcha de forma automática, con determinadas excepciones para el caso de circunstancias excepcionales.

Estos objetivos de déficit estructural deben estar incorporados a los ordenamientos de los estados obligados, preferentemente en norma de rango constitucional y monitorizado por instituciones independientes. El no cumplimiento por los países obligados a ello, de la incorporación de reglas de estabilidad presupuestaria en los términos exigidos por el Tratado, podría dar lugar a sanciones de hasta $0.1 \%$ del PIB del país incumplidor, que serían destinadas, en el caso en que fuese un país del área del euro, al European Stability mecanismo y en otro, al presupuesto de la Unión.

Al margen de estas previsiones, el TECG refuerza el PEC (reformulación de la regla sobre deuda pública, y regla de voto mayoritario cualificado inverso para la zona euro cuando la Comisión estime que hay una situación de déficit excesivo), y establece elementos básicos de refuerzo de la vigilancia y coordinación de las políticas económicas. Así, desarrolla mecanismos de coordinación ex/ ante de planes y programas de emisión de deuda publica entre los países y programas de colaboración con los países en situación de procedimiento de déficit excesivo, que incluyen el diseño de las medidas de reforma estructural que se consideren convenientes para que la salida de aquella situación sea efectiva y perdurable en el tiempo.

Por último, en el aspecto de la gobernanza económica de la EZ, se incluyen previsiones como las Cumbres de la ZE, a celebrarse al menos dos veces al año, y procedimientos de cooperación económica reforzada.

Un elemento que facilitó poner en marcha este tratado, fue sin duda el hecho de que desde el primer momento los estados firmantes acordaron eliminar la unanimidad para la entrada en vigor de este tratado el $1^{\circ}$ de enero de 2013. 
En opinión de Carlos Closa (2012) ${ }^{7}$ investigador del Instituto El Cano, hay tres aspectos adicionales de este procedimiento o asociados con el mismo que tienen importancia sustantiva de cara a la ratificación de este tratado:

1. Consolida la existencia de un núcleo duro dentro de la UE, ya que aquella depende explícitamente del consentimiento de los miembros del euro a pesar de que el Tratado pueda ser ratificado por los otros EM de la UE

2. En cuanto al ámbito de aplicación, ya que se aplicara únicamente a los estados que hayan ratificado. Esto podría percibirse como un poderoso incentivo para desertar dado que el TECG impone obligaciones sustanciales a sus firmantes ("la regla de oro")

3. Las adhesiones posteriores: los no firmantes (RCH y RU) pueden, de acuerdo con el artículo 15, adherirse posteriormente.

Aunque estas posibilidades no se presentasen como una cuestión inmediata, los problemas que puede presentar no son menores, en particular, al tener en cuenta el "cerrojo" creado por la European Union Act (2011), mediante la cual... "los gobiernos británicos necesitaran un referéndum para ratificar cualquier tratado que implique una cesión de soberanía” (como el TECG). Para satisfacer el requisito de fusión con el TUE, se podría optar por realizarse sin incorporar previamente al Reino Unido al TECG. Pero puesto que la fusión requiere en cualquier caso una revisión mediante el procedimiento del artículo 48 (es decir, la unanimidad) y que cualquier gobierno británico necesitara un referéndum, es obvio que subsisten graves problemas de cara al futuro para amalgamar ambas estructuras en el mismo tratado.

Para mejorar la competitividad y evitar desequilibrios se optó por un euro plus mismo que lograría en el mediano y largo plazo conseguir mayor empleo, competitividad, sostener unas finanzas públicas saneadas y por añadidura un sector financiero estable.

El pacto no solo puso en pie la unión económica que junto a la monetaria velaran por el crecimiento al crear empleo, sino apostó por frenar el despilfarro y luchar porque el crédito fluya a familias y empresas, de este modo apuesta por un sistema económico más competitivo tanto en los diferentes mercados como en los servicios.

En línea con la Estrategia Europa 2020 y con la idea fija de estabilizar el euro se crea lo que se denominó Semestre Europeo para cumplir con los siguientes objetivos:

${ }^{7}$ www.realinstitutoelcano.org/wps/portal núm. 96, abril 2012. 
1. Mejorar la coordinación de las políticas económicas

2. Integrar todos los instrumentos de supervisión (fiscales y macroeconómicos)

3. Hacer recomendaciones coherentes a cada uno de los estados miembros para la elaboración de su presupuesto y definir su política económica.

Para lograr estos objetivos se consideró como elementos clave:

1. La elaboración de un estudio prospectivo de crecimiento. Es un informe que analiza los objetivos para toda la UE. De este informe se derivan los ejes prioritarios que habrán de perseguirse en cada ocasión, por ejemplo en el año 2014, los ejes son:

- Consolidación fiscal

- Restablecer el crédito a la economía

- Promover el crecimiento y la competitividad

- Afrontar el desempleo y las consecuencias sociales de la crisis

- Modernizar la administración pública

2. Proyectos nacionales de regiones y programas de estabilidad, crecimiento y convergencia. Son documentos que elabora cada estado miembro para informar sobre las reformas que piensa realizar en el año siguiente

3. Una vez revisados estos proyectos, se hacen recomendaciones específicas a cada EM, por tanto, son de aplicación nacional

El ciclo del "Semestre Europeo 2014" dio comienzo el 13 de noviembre de 2013 con la publicación de los dos informes preceptivos de la Comisión:

1. El "Informe sobre el Mecanismo de Alerta de Desequilibrios Macroeconómicos 2014”, que reclamaba, en esta ocasión la realización de “exámenes exhaustivos" sobre $17 \mathrm{EM}$

2. La "Encuesta Anual sobre el Crecimiento 2014", que establecía las prioridades de la UE para impulsar el crecimiento y la creación de empleo, y que debía condicionar los contenidos de los planes Nacionales de 2014, los cuales serían objeto del análisis comunitario en las "Recomendaciones específicas por país".

1 ) Concretamente el Informe señalaba la presencia o no de Desequilibrios en los 17 EM de la EZ, señalando en esta ocasión: 
3 Países sin: Dinamarca, Luxemburgo y Malta

14 con: Bélgica, Bulgaria, Alemania, Irlanda, España, Francia, Croacia, Italia, Hungría, Holanda, Eslovenia, Finlandia, Suecia y Reino Unido.

3 de estos con desequilibrios excesivos: Croacia, Italia y Eslovenia.

Este informe insistía en la persistencia de la vulnerabilidad y que el ajuste está lejos de haber concluido.

El alto endeudamiento tanto privado como público sigue planteando riesgos para el crecimiento y para la estabilidad financiera.

El desempleo se mantiene en niveles alarmantes y la recuperación de la competitividad internacional tendrá que mantenerse si se quiere reducir la deuda externa.

2) Encuesta Anual de Crecimiento, 2014: en abril, los EM presentaron sus planes para conseguir unas finanzas públicas saneadas (Programas de Estabilidad o Convergencia), así como las reformas y medidas que se necesitan alcanzar para un crecimiento inteligente, sostenible e integrador ${ }^{8}$ en áreas como el empleo, la investigación, la innovación, la energía o la integración social (denominados planes nacionales de Reforma).

Siguiendo con la secuencia prevista en el Semestre Europeo, el 2 de junio, la Comisión publicó las "Recomendaciones Específicas" para cada país tras evaluar los programas y planes nacionales. En cada uno de los países hizo hincapié en las acciones que deben seguir para lograrlo.

\section{Conclusiones}

Este ensayo enmarca el hecho de que son muchos los esfuerzos que se han desplegado para apuntalar la UEM. Empezando con el reconocimiento de haber creado una Unión Monetaria sin establecer paralelamente un gobierno económico en aquel tiempo. Entonces se pensó que se podría salir del paso con un poco de suerte y unas cuantas advertencias disuasorias.

En los hechos se creó una arquitectura " provisional" sustentada en tres pilares, a saber:

$\left.1^{\circ}\right)$ Una política monetaria centralizada de carácter federal. Encomendada a un banco independiente, el BCE, diseñada a imagen y semejanza del Bundesbank

${ }^{8}$ Es el tipo de crecimiento que persigue la Estrategia para el año 2020-2030. 
$2^{\circ}$ ) Un PEC diseñado para frenar los excesos presupuestarios de los PM

$\left.3^{\circ}\right)$ Una coordinación "ligera" (sin incentivos ni sanciones) del resto de las políticas económicas.

Estos tres pilares se completaron con tres advertencias: $\mathrm{NO}$ rescate, $\mathrm{NO}$ insolvencia, NO salida.

Sin embargo, mucho antes de que estallase la crisis financiera, esta arquitectura provisional empezó a mostrar serias grietas que al principio no se notaron porque el dinero corría sin tasa, no obstante, permanecían en cada uno de los tres pilares:

- La fijación de unos tipos de interés únicos para países en diferente fase del ciclo económico y que mostraban elevados diferenciales de inflación provocaron el desplazamiento del ahorro de las economías centrales a las periféricas, alimentando así las burbujas de estas últimas

- El PEC se incumplió sistemáticamente y lo que es más grave, cuando fue incumplido por Francia y Alemania se decretó una amnistía general y un cambio de las reglas del juego

- La coordinación ligera de las políticas económicas no funcionó, siendo las diferencias a los cinco años de empezando el proceso, más fuertes que en el momento de su lanzamiento

- La regulación y la supervisión del sistema financiero se evidenciaron inadecuadas.

De esta forma, cuando llego la crisis, los pilares sobre los que se sostenía la moneda única empezaron a resquebrajarse y el edificio empezó a cuartearse peligrosamente: el BCE no contaba con los instrumentos para financiar la economía europea ni para atajar los ataques contra la deuda soberana de alguno de los EM. Su protagonismo en la salida de la crisis y en la recuperación económica fue mucho más tímida que la actuación de la Reserva Federal o la del Banco de Inglaterra. 11 de 17 países de la Ez superaban los límites establecidos por el PEC.

La coordinación económica fracasó como demuestra el hecho de que Alemania pudiese colocar sus bonos prácticamente gratis, mientras que el bono griego llego a estar 2532 puntos por encima del bono alemán a diez años. Algo que no ha ocurrido nunca en ninguna de las uniones monetarias que la historia ha conocido. Y, las tres advertencias disuasorias, que pretendían salvaguardar la unión monetaria, saltaron también por los aires: hubo necesidad 
de tres rescates, se estuvo a nada de una insolvencia casi total y el riesgo de que Grecia abandonara la Ez.

Para evitar que el edificio se derrumbara, se adoptaron dos tipos de actuaciones de urgencia:

1. Rescatar a las economías más amenazadas e

2. intervenir en los mercados de deuda para frenar la especulación

Las crisis de solvencia de Grecia, Portugal e Irlanda exigieron el establecimiento de mecanismos de solidaridad financiera (primero, el establecimiento del Fondo de estabilidad económica financiera, FEEF) y posteriormente el Mecanismo... MEDE, ambos sirvieron de cortafuegos a la crisis de los mercados de deuda pública. Cortafuegos que nacieron lastrados porque casi todas las decisiones tienen que ser adoptadas por unanimidad y porque la pólvora de que disponen es más bien escasa cuando se trata de contrarrestar una especulación mundial de una potencia de fuego prácticamente ilimitado.

La adopción por el BCE del Outright Monetary Transactions, ${ }^{9}$ en septiembre de 2012, tras las decisiones del Consejo de junio, ha sido muy relevante. Este mecanismo permite la intervención del BCE en los mercados secundarios de deuda de títulos emitidos por aquellos EM que cumplen con los compromisos asumidos en los procedimientos de gobernanza económica, lo que, sin haber llegado a ser utilizado, ha resultado clave para poner fin a la volatilidad de los mercados de deuda y ha contribuido a una mejor transmisión de la política monetaria.

Junto a estas actuaciones de urgencia, en el año 2013 se adoptaron otras medidas de carácter más estructural: las destinadas a fortalecer la Ez en sus aspectos fiscales y económicos y las diseñadas para construir una Unión Bancaria Europea ${ }^{10}$ que acabase con la fragmentación de los mercados bancarios

\footnotetext{
${ }^{9}$ La intervención del BCE se ha materializado en un programa de compra masiva de deuda pública en el mercado secundario, llamado Compras Monetarias Directas (Outright Monetary Transactions, OMT por sus siglas en inglés). El BCE es una renovación del anterior programa de compra de bonos llamado SMP (Securities Markets Program) instaurado en mayo de 2010 tras la crisis griega.

${ }^{10}$ La unión bancaria es el proyecto europeo más ambicioso que se ha acometido desde la introducción de la moneda única. Se emprendió en el verano de 2012 con el fin de enviar un mensaje de unidad frente al problema de fragmentación financiera que estaba poniendo al euro contra las cuerdas. El objetivo principal se basa en reanudar el progreso hacia el mercado único de los servicios financieros y a un nivel más general, preservar el mercado único europeo. Al resituar a Europa en la vía correcta para la integración, la unión bancaria restablecerá el impulso hacia una auténtica unión económica y monetaria.
} 
y las distorsiones que se derivan de las diferencias de los tipos de interés así como también romper el vínculo entre deuda bancaria y deuda soberana.

Como se ha expuesto en este ensayo, en el ámbito fiscal se aprobó el reforzamiento del PEC a través del paquete fiscal y de los llamados Six Pack (que, en grandes líneas, fortalece la capacidad sancionadora de la Comisión a través de la llamada mayoría cualificada inversa) y el Two pack (que exige a los Gobiernos el envío a la Comisión de los anteproyectos de presupuestos nacionales antes de la remisión a los Parlamentos nacionales).

En el ámbito económico se aprobó un PDM que establece un procedimiento de seguimiento de una serie de indicadores macroeconómicos y de sanción en caso de desviaciones.

Ya se cuenta con una autoridad bancaria europea. Se avanzó en la aprobación del MUS que ejercerá el BCE de manera directa para las entidades consideradas significativas, el MUR que deberá configurarse con una autoridad única y un Fondo común de resolución que garanticen el tratamiento similar a las entidades de crédito.

Aunque las medidas que se han tomado significan avances importantes, es necesario seguir haciendo más si se desea realmente garantizar la sostenibilidad de las Finanzas Públicas. Por ejemplo, se debería atribuir a las Autoridades de la UE el derecho a revisar los presupuestos nacionales en casos de desviación de la disciplina presupuestaria previamente acordada en la UE. Ello exigiría modificar la naturaleza de los dictámenes previstos en el Two pack que deberían pasar de no vinculantes a vinculantes.

Los acuerdos entre EM e Instituciones que aseguren la convergencia dentro de la UEM, deben estar aparejados con mecanismos de solidaridad que apoyen las reformas y favorezcan la inversión, el crecimiento y la generación de empleo. Para crecer y acelerar ese crecimiento el BCE y el BEI deben facilitar el crédito.

En el camino hacia una solidaridad financiera se debería crear un Fondo Monetario Europeo (FME), al que solo accederían los países que cumplieran el paquete de gobernanza. Este fondo tendría entre sus funciones:

1. ejercer los poderes atribuidos hoy al MEDE

2. emitir eurobonos mancomunados y bonos para proyectos de interés europeo.

Obviamente fortalecer la UEM no agota los cambios que hay que hacer, se necesita aumentar la capacidad de actuación interna y externa para lograrlo. 


\section{Bibliografía}

Aldcroft, Derek, H., Historia de la economia europea 1914-2000, ed. Ampliada y actualizada, 2012. Ed. Crítica Editorial, S.A., Madrid.

Bilbao Ubillos, Javier, "Gobernanza europea de la crisis, una visión crítica: racionalidades inherentes a las estrategias de salida" XI Premio Francisco Javier de Landaburu Universitas, EUROBASK, febrero 2013.

Closa, Carlos, "Jugando a ratificar: las reglas de entrada en vigor del nuevo Tratado de Estabilidad, Coordinación y Gobernanza del Euro", www.realinstitutoelcano.org/wps/portal.

Domínguez Domech, Carmen (Directora) y Juan Manuel Menéndez Blanco, “Crisis económica y financiera. La respuesta de la UE: Una evaluación de las políticas aplicadas y un análisis de las ineficiencias creadas", viII Premio Francisco Javier de Landaburu Universitas, EUROBASK, diciembre 2009.

Iñarritu, Beatriz, Actualidad institucional y económica de España en el marco de la Unión Europea, Cuadernos Económicos de Deusto, Núm. 51, 2014, Bilbao, pp. 185-206.

web Instituciones Europeas (Comisión Europea, Parlamento, Consejo). 